BOOKS

\title{
Should you or shouldn't you? Guidance on the HPV vaccine
}

The HPV Vaccine Controversy: Sex, Cancer, God, and Politics. A Guide for Parents, Women, Men, and Teenagers Shobha S. Krishnan MD

Praeger Publishers; 2008.

248 pp $\$ 39.95$

I $\mathrm{n}$ the past, vaccines have played a major role in reducing the threat of feared diseases such as polio and smallpox. Today, two new HPV vaccines have been touted as a public health breakthrough in helping to prevent another feared disease, cervical cancer, and in helping to significantly reduce a prevalent sexually transmitted infection, genital warts.

In The HPV Vaccine Controversy: Sex, Cancer, God, and Politics, Dr. Shobha S. Krishnan describes in a detailed yet understandable manner how cervical cancer and anogenital warts are caused by the human papillomavirus (HPV). She presents recent epidemiological data that indicate more than 20 million people in the United States already have HPV and 6.2 million new cases are diagnosed every year. She also discusses the health and social consequences related to HPV and presents a thorough review of the HPV literature.

Seventy per cent of cervical cancer is caused by two strains of HPV, 16 and 18 , and 90 per cent of anogenital warts are caused by the two strains of HPV, 6 and 11 , all four of which potentially can be prevented by the quadrivalent HPV vaccine. So, if this vaccine is safe and effective in preventing harm, why in the United States does there exist an HPV vaccine controversy?

The answer to the above question, and many more frequently asked questions about HPV, are discovered in the author's thorough presentation of the

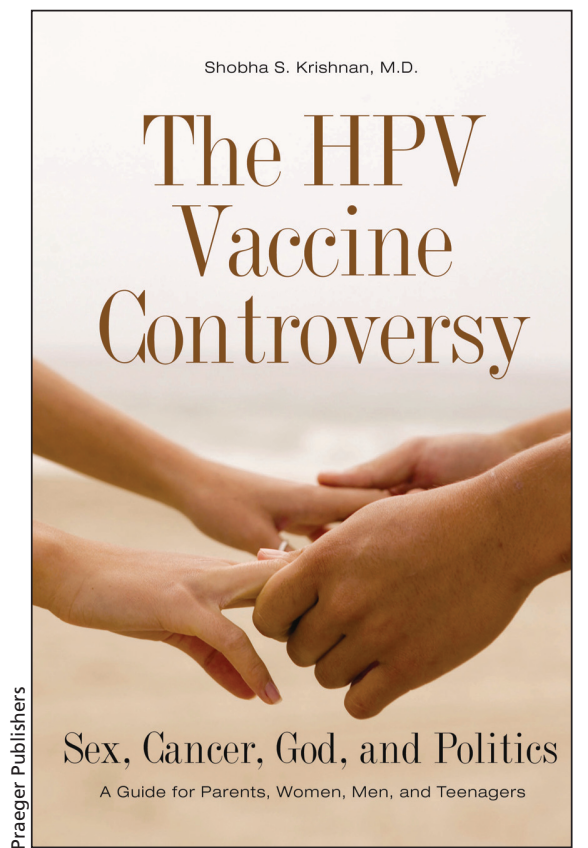

science, social and ethical issues that surround the recommendations made by the Centers for Disease Control and Prevention to vaccinate young girls between the ages of 11 and 12. Many countries, including Canada, have done the same and Australia has included boys as well. The goal is to administer the vaccine before young people become sexually active.

Krishnan presents evidence that the HPV vaccine is a cost-effective intervention and its implementation will uphold the principle of harm reduction and benefit the common good, especially among socio-economically disadvantaged girls who experience disproportionate higher rates of cervical cancer.

The book is dominated by relevant health and medical information to help the reader decide whether this is a scientifically and ethically sound diseaseprevention strategy.

The controversy over the use of an HPV vaccine pits ideology against sci- entific advancement. Krishnan points out that opponents to vaccination may believe that exposing children to an HPV vaccine will increase sexual activity among youths, undermine family values forbidding premarital sexual relationships and promoting abstinence, and create a false sense of security about being protected from sexually transmitted infections. As a result, even though science offers evidence to support the safety and effectiveness of HPV vaccines, opponents believe government should not intrude upon individual choices about medical interventions and sexual behaviour. Meanwhile, proponents of the vaccine, argue that it "will irrefutably reduce the occurrence of the second leading cause of cancer in women around the world."

One of the great strengths of the book is the author's use of pedagogical techniques, such as "Myths and Facts" and "Key Facts" sections in each chapter.

Krishnan obviously wants the reader to make up his or her own mind about the controversy, however, on page 170 she does opine that "it is worth vaccinating girls and young women to reduce the physical, psychosocial, and economic burden of HPV infections."

The subtitle includes A Guide for Parents, Women, Men, and Teenagers. However, this book is also an excellent resource for health professionals; it is a fact-based and balanced approach to the HPV vaccine controversy and achieves her aim of having "the reader become well-informed and educated with the disease manifestations of HPV and the roles of the new vaccines."

\section{Joseph E. Balog MD}

Department of Health Science Brockport, NY

Competing Interests: None declared. 